\title{
Pitou type probe for flow velocity registration with possibility of independent temperature control
}

\author{
A.G. SEMENOV
}

N.N. Andreev Acoustics Institute, Russian Academy of Sciences, 4 Shvernik str., Moscow 117036, Russia

\begin{abstract}
Proposed control equipment scheme is based on acoustic Helmholtz resonator property to change its resonance frequency when sound speed in the vicinity of its throat inlet is changed. So this kind of resonator with pressure measuring acoustic transducer is installed in well known Pitou probe static pressure volume envelope, slightly changing its construction.

To use Pitou probe of new type, except of difference between dynamic and static pressure conventional measurement, one must additionally control acoustic pressure spectrum maximum frequency observed in resonator volume by means of spectrum analyzer or other frequency measuring device connected to transducer.

Graduation curves for Helmholtz resonator frequency are presented showing that resonance frequency change is linearly dependent on sound speed change and - by square root law - on temperature change.

Initial experimental results conducted in model circumstances (in air) are presented giving possibility to estimate effect of measuring equipment proposed.
\end{abstract}

\section{INTRODUCTION}

Acoustic measurements in realistic situation, for instance, in ocean, encounter many problems due to environmental currents. Moreover, ocean medium is stratified, its parameters during measuring process could be tremendously changed due to change of temperature, density or salinity. Anyway, sound speed in the medium surrounding measuring point could be changed and acoustic field measurements can give erroneous results. That is why it is desirable to control flow velocity and sound speed values constantly, especially measuring in complex environmental conditions occurring in ocean.

New Pitou type probe could be related to control equipment used in acoustics measurement for environment parameters control. It could be used, for instance, for control of flow velocity or temperature in the vicinity of acoustic transducer. One of widely known means measuring flow velocity is thermoanemometer, including thermoresisting wire placed in flow [1], but a row of problems arises in experimental practice due to change of thermoanemometer parameters in time connected to process of its fast deterioration. Moreover, conventional thermoanemometers are very expensive and not strong enough to be widely used in ocean experiment. Other widely known way of flow velocity control is to use conventional pitou tube designed of cylindrical housing with input 
inlets of total and static pressures situated in the center of end and on cylindrical housing surfaces and communicated with corresponding cavities with pressure transducers [1]. This device is very convenient and reliable in experiment, but to use its data properly additional information on temperature of flow is necessary. Usually this information is obtained from temperature probes situated in the vicinity of Pitou tube velocity probe. Their spatial position frequently differs substantially and accuracy of resulting environmental parameters control could be lessen this way. This well known technical contradiction could be solved in special Pitou type probe construction designed to take into account velocity and temperature fluctuations encountered in experiment simultaneously.

\section{PROBE DEVELOPMENT}

Aim stated above is reached by changing construction of static pressure cavity of known Pitou tube [3]. It is designed in the form of acoustic Helmholtz resonator, where static pressure inlet coincides with inlet of resonator. Schematic construction of sound speed and temperature fluctuations protected Pitou type probe for flow velocity registration is shown on Fig.1. Proposed transducer comprises: cylindrical housing 1 with inlet 2 of total pressure situated in the center of end housing surface, static pressure inlet 3 on its cylindrical surface. Inlets 2 and 3 are communicated with cavities of total (not shown) and static 4 pressures correspondingly, connected with pressure transducers not shown on the scheme by channels 5 . Housing 1 is additionally supplied with acoustic resonance temperature probe coinciding with static pressure cavity 4. On its wall acoustic pressure transducer 6 is placed.

Velocity measuring device of the type proposed is working in following manner. When probe housing 1 is streamlined by flow, pressures in the inlets 2 and 3 are equal to total and static pressures correspondingly. These pressures are measured by pressure transducers installed in the probe. Simultaneously pressure pulsation of flow stimulates acoustic vibration in cavity 4 of acoustic resonator with resonance frequency of vibrations depending on sound velocity or temperature value in the vicinity of static pressure inlet 3 . Inlet tube is designed with crossection increasing in direction to the center of housing to lessen nonlinear losses taking place in flow acoustic vibrations. Development of new Pitou type probe should be supported by physical analysis of situation in the flow.

\section{PHYSICAL ANALYSIS}

The problem to be analyzed is the behavior of pressure probe at rest in surrounding flow. If probe would move with fluid, then true static pressure $p_{s}$ and acoustic pressure $p_{a}$ would be measured in both cavities. If the same transducer is kept at rest, however, so that there is relative motion between it and fluid, then quantities measured in transducer inlets 2 and 3 could differ from $p_{s}$ and $p_{a}$. Let us suppose, that pressure measured by transducer moving with flow, is $p_{s}$, then at

the openings 2 and 3 we should have [1]

$$
\mathrm{p}_{2}=\mathrm{p}_{\mathrm{s}}+\frac{\gamma-1}{\gamma} \frac{\rho \mathrm{v}^{2}}{2}, \mathrm{p}_{3}=\mathrm{p}_{\mathrm{s}}
$$


where $\rho$ and V - fluid density and velocity correspondingly, $\gamma$ - specific heats ratio. It is important to notice, that in practice $\rho$ and $\gamma$ could be temperature dependent, that is why additional temperature control in newly designed Pitou tube is provided.

In the presence of a plane incident wave with acoustic pressure $p_{a}$ and fluid velocity $u$, in the field away from the probe $p_{s}=p_{0}+p_{a}, \rho=\rho_{0}+\delta$ and $\mathbf{v}=\mathbf{v}_{0}+\mathbf{u}$. If wavelength of sound is much longer than probe dimensions, pressures $\mathrm{p}_{2}$ and $\mathrm{p}_{3}$ could be expressed in the form

$$
p_{2} \quad p_{0}+p_{a}+\frac{\gamma-1}{2 \gamma}\left(p_{0}+\delta\right)\left(v_{0}+u\right)^{2}, p_{3}=p_{0}+p_{a}
$$

Corresponding acoustic pressures $p_{2 a}$ and $p_{3 a}$ measured in total and static pressure cavities are

$$
p_{2 a}=p_{a}\left(1+\frac{\gamma-1}{\gamma} M_{0} \cos \varphi\right), p_{3 a}=p_{a}
$$

Thus static pressure inlet 3 measures acoustic pressure the same, as transducer moving with flow. If turbulent fluctuations of mean velocity $u$ much smaller than $v_{0}$ take place in flow, then corresponding turbulent pressure field of the order of $\rho u^{2} / 2$ acts on inlet 3 driving acoustic oscillations on resonance frequency of static pressure cavity 4. If fluctuation velocity $u$ is too small to drive acoustic resonator, then one additional possibility could be used. Practically always velocity measurements with small enough probe are fulfilled in flows of Reynolds number calculated on the basis of housing transverse dimension $d$ laying presumably in the interval $10^{2}-10^{5}$, where fluctuations of oncoming flow are practically periodic in time with frequency of the order $0.2 \mathrm{~V} / \mathrm{d}$. Mentioned above acoustic resonator could be preliminary adjusted or designed with resonance frequency close to expected in flow. If in conventional case, when Karman vortex street is not generated, stronger fluctuations are observed on inlet 2 oriented contrary to the flow direction, in mentioned above case, inlet 3 is more profitable. Typical averaged narrowband spectra of sound noise processes recorded in heated air flow by acoustic pressure transducer 6 installed in resonator 4 for two values of flow temperature $T_{1}=20^{\circ} \mathrm{C}$ and $T_{2}=100^{\circ} \mathrm{C}$ are shown on Fig.2 together with typical calibration resonance frequency sound velocity (temperature) dependence shown on Fig.3 [2]. Resonance frequencies could be easily determined from this narrowband spectra to be $f_{1}=2210 \mathrm{~Hz}$ and $f_{2}=2480 \mathrm{~Hz}$. It is useful to remember, that resonance frequency of Helmholtz resonator designed in static pressure cavity is proportional to sound velocity value or square root of temperature, as well as square root of inlet 3 crossection and inversely proportional to square root of cavity 4 volume and inlet 3 length product. For dimensions of cavity 4 are known, temperature or sound velocity in the vicinity of inlet 3 opening could be easily calculated by acoustic pressure spectrum measurements. Knowing temperature, one could estimate using expressions cited above real environment constant and turbulent velocities of flow, which could give rise to corresponding artificial pressure signals in ocean experiments.

\section{REFERENCES}

[1] Morse P and Ingard U, Theoretical acoustics, 1968, monograph, N.Y.-London, MCGrow-Hill publishers, pp.702-704. 
[2] Gromov Yu I, Semenov A G et al, Resonator in finite amplitude sound wave field, 1977, Akustichesky zhurnal, vol. 23, no 1, pp.160-162 (in Russian); Soviet Physics-Acoustics, 1977, vol.23, no 1 (in English).

[3] Gromov Yu I, Semenov A G et al, Gas flow velocity probe, official USSR Bulletin of Inventions, Samples and Trade-marks, 1978, no 13, description of USSR patent no 601616 , claimed 25.12.76.



Fig.l. Schematic construction of sound speed and temperature fluctuations protected Pitou type probe for flow velocity registration.

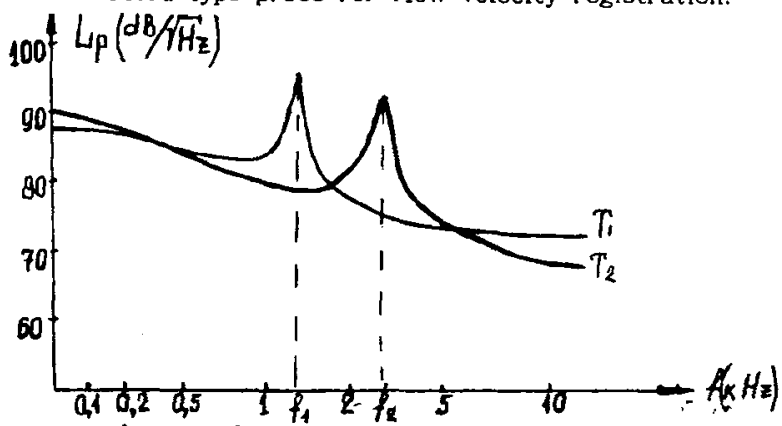

Fig.2. Typical averaged narrowband spectra of sound noise processes recorded in heated air flow by pressure transducer installed in resonator for two values of flow temperature $T_{1}=20^{\circ} \mathrm{C}$ and $T_{2}=100^{\circ} \mathrm{C}$. Corresponding resonance frequency values $f_{1}=2210 \mathrm{~Hz}$ and $f_{2}=2480 \mathrm{~Hz}$. Spectrum level $\left(\mathrm{dB} / \mathrm{Hz}^{1 / 2}\right)$, frequency $(\mathrm{kHz})$.

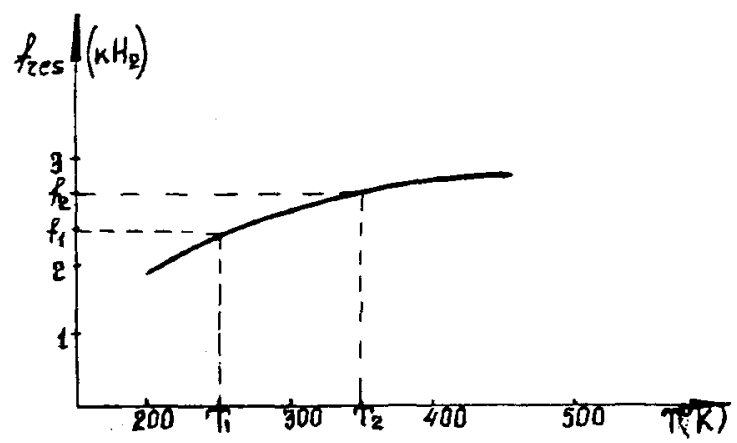

Fig.3. Typical calibration resonance frequency sound velocity (temperature) dependence for heated air flow. Resonance frequencies and temperatures $f_{1}, T_{1}$ and $f_{2}, T_{2}$ are the same as on Fig.2. Frequency $(\mathrm{kHz})$, temperature $\left({ }^{\circ} \mathrm{K}\right)$. 